\title{
BREAST IMAGING
}

\section{Can strain elastography be used in reclassification of indeterminate breast lesions in BIRADS lexicon?: A prospective} study

\author{
Dimpi Sinha, Nischal G Kundaragi', Sukrity Sharma, Sudhir K Kale \\ Departments of Radiology and 'Interventional Radiology Aster CMI Hospital Bangalore, Karnataka, India \\ Correspondence: Dr. Dimpi Sinha, Aster CMI Hospital, Airport Road, Bangalore - 560 092, Karnataka, India. E-mail: drdimpisnh@gmail.com
}

\begin{abstract}
Purpose: To evaluate the role of strain elastography (SE) in reclassification of indeterminate breast lesions placed under BIRADS 3 and 4 categories by conventional ultrasound (US) parameters so as to recourse biopsy only for suspicious stiffer lesions. Methods: 113 breast lesions in 100 women assigned as BIRADS category 3 and 4 on US parameters were prospectively evaluated by SE followed by histo-pathological examination. Strain ratio (SR) and Elastography Score (ES) were determined for each lesion. The sensitivity, specificity, accuracy, positive predictive value (PPV) and negative predictive value (NPV) were calculated for each modality and diagnostic performance were compared. The best cut-off point was calculated for each of the elastography parameter using the receiver operator curve analysis (ROC). Results: Out of the 113 lesions, 40 were malignant (35.4\%) and 73 were benign (64.6\%). A statistically significant difference was observed in the AUC for ES and conventional US: 0.98 vs 0.90 (Difference $=0.08, P=0.02$ ). Elastography parameters were more specific as compared to US (ES-94.5 \& SR-93.2\% vs $63 \%$, $P<0.05$ ) with high NPV. SE performed better in BIRADS 4 category lesions. On the basis of elastography parameters, $85 \%$ of BIRADS 4 category lesions were correctly predicted as benign with overall sensitivity, specificity and diagnostic accuracy of elastography being $97 \%, 84.6 \%$ and $91.9 \%$. Among BIRADS 3 category lesions, 97\% were correctly predicted as benign and $50 \%$ lesions were correctly predicted as malignant with a sensitivity of $50 \%$, specificity of $97.8 \%$ and diagnostic accuracy of $94 \%$. Conclusion: Strain elastography is a useful adjunct to conventional ultrasonography for the assessment of indeterminate breast lesions and may help in avoiding unnecessary follow ups and biopsies. Elastography score is a better parameter as compared to strain ratio.
\end{abstract}

Key words: Breast cancer; elasticity score; strain elastography; strain ratio; ultrasonography

\section{Introduction}

Breast cancer is the most commonly diagnosed cancer among women globally with approximately 2 million new cases in 2018. ${ }^{[1]}$ It is also the leading cause of cancer death among women worldwide with an estimated

\begin{tabular}{|l|l|}
\hline \multicolumn{2}{|c|}{ Access this article online } \\
\hline Quick Response Code: & \multirow{2}{*}{ Website: } \\
\cline { 1 - 2 } & www.ijri.org \\
\cline { 1 - 2 } & DOI: \\
& 10.4103/ijri.IJRI_425_19 \\
\hline
\end{tabular}

626,679 deaths in 2018. ${ }^{[1]}$ Breast cancer is the most common cancer among Indian females with age adjusted rate as high as 25.8 per 100,000 women with high mortality

This is an open access journal, and articles are distributed under the terms of the Creative Commons Attribution-NonCommercial-ShareAlike 4.0 License, which allows others to remix, tweak, and build upon the work non-commercially, as long as appropriate credit is given and the new creations are licensed under the identical terms.

For reprints contact: WKHLRPMedknow_reprints@wolterskluwer.com

Cite this article as: Sinha D, Kundaragi NG, Sharma S, Kale SK. Can strain elastography be used in reclassification of indeterminate breast lesions in BIRADS lexicon?: A prospective study. Indian J Radiol Imaging 2020;30:493-9.

Received: 15-Nov-2019

Accepted: 11-Aug-2020

Revised: 16-Jan-2020 Published: 13-Jan-2021 
about 12.7 per 100,000 women. ${ }^{[2]}$ Early detection is the key for successful management of breast cancer. Various screening programs incorporating mammography and ultrasonography are being run worldwide, for the early detection of breast cancer. Using the standard American College of Radiology (ACR) Breast Imaging and Reporting Data System (BIRADS) lexicon, the lesions are placed in different categories, based on which clinical actions are taken. Most of the indeterminate lesions categorized into BIRADS 3 or $4 \mathrm{~A}$ categories pose a diagnostic dilemma for the treating clinician and the reporting radiologist. They are low suspicion lesion with a reported incidence of malignancy as $<10 \%$. About $98 \%$ of the lesions, graded as BI-RADS 3, are histologically benign; the current guidelines suggest short-term follow-up. Nevertheless, about $2 \%$ of these lesions eventually turn out to be malignant, which are missed at the initial diagnosis. Compliance with regular follow-up for BIRADS 3 lesions is poor, thereby prompting clinicians for biopsy over regular follow ups, which in most of the cases turn out to be negative. BI-RADS 4 lesions have a low-to-moderate probability of malignancy (2-94\%), and biopsy is recommended. Among BI-RADS category 4 A lesions, approximately only $2-9 \%$ turn out to be histologically malignant, and a much larger proportion of patients undergo invasive diagnostic procedures that could be avoided if a better noninvasive imaging technique was available for accurate diagnosis.

Conventional ultrasound is the most widely used and universally accessible modality for the evaluation of breast lesions. The relatively lower specificity of breast ultrasound has led to new developments in sonographic technology, and elastography is the most important technique to improve lesion characterization in breast ultrasonography based on lesion stiffness.

A prospective study was designed in our institute to determine whether strain elastography (SE) could downgrade or upgrade BI-RADS 3 and 4 lesions, thereby recoursing biopsies only to suspicious stiff lesions.

\section{Methods}

The study was approved by the Institutional Review Board, and written informed consent was obtained from all participants before being included in the study. A total of 113 lesions in 100 patients, referred for conventional breast ultrasound, were analyzed from August 2018 to June 2019 at the Department of Radio-diagnosis in our hospital.

Real-time ultrasound followed by SE was performed using a 3-12 MHz linear array transducer on a Samsung RS80A unit (Samsung Medison BLDG., 42 Teheran-ro 108-gil, Gangnam-gu, Seoul 135-851, South Korea) by one of the two radiologists with 8 and 10 years of experience in breast ultrasounds and training in elastography.
Patient selection

Females with sonographically visible solid breast lesions, measuring less than $3 \mathrm{~cm}$, classified as BI-RADS 3 and 4 on conventional ultrasound were included in the study. Cystic lesions, solid lesions classified as BIRADS category 2 or 5 , lesions located near the skin surface or the chest wall or those lesions without cytologic/histopathologic diagnosis were excluded from the study.

\section{Conventional sonography}

The lesions were first assessed by conventional B-mode ultrasonography using a radial scanning pattern with patients lying in a supine position. Each lesion was assigned a BI-RADS category using conventional ultrasound features like shape, echotexture, margin, orientation, and posterior acoustic characteristics.

\section{Elastography technique and parameters}

Next, SE was performed. Data were acquired by setting the field-of-view box including the region from the subcutaneous fat layer to the pectoralis muscle layer, avoiding the rib cage. Due care was taken to include the entire lesion within the field of view. The target lesion was vertically compressed with application of optimum light external pressure to the transducer (an adequate probe pressure on the target lesion was displayed as two or three blocks of green in the vertical column on the left side of the monitor of the ultrasound scanner; a partially adequate pressure was displayed as single or no block of color and high levels of pressure displaying as four to five blocks).

The Elastography score (ES) was determined on a five-point Tsukuba classification proposed by Itoh et al. ${ }^{[3]}$ According to Tsukuba classification, a score of 1 is given when the whole lesion is evenly shaded in green, indicating that the entire lesion is soft with homogeneous strain throughout [Figure 1]. A score of 2 is represented by a mixed pattern of green and blue suggesting that the greater part of the lesion is soft with a few interspersed areas of stiffness [Figure 2]. A score of 3 is given when the lesion shows strain at the periphery represented by green shade, with central stiffness represented in blue [Figure 3]. A score of 4 is given when the lesion shows homogeneous shading in blue indicating that the entire lesion is stiff [Figure 4]. Finally, a score of 5 is given when the entire lesion and surrounding area shows blue shading indicating stiffness in and around the lesion [Figure 5]. Lesions with ES 1-3 were considered benign, and lesions with ES 4 and 5 were suspected to be malignant.

Strain ratio (SR) was calculated by placing first the region of interest (ROI) in target lesion and second ROI in lateral subcutaneous fat tissue of similar size and at the same depth as the target lesion. 


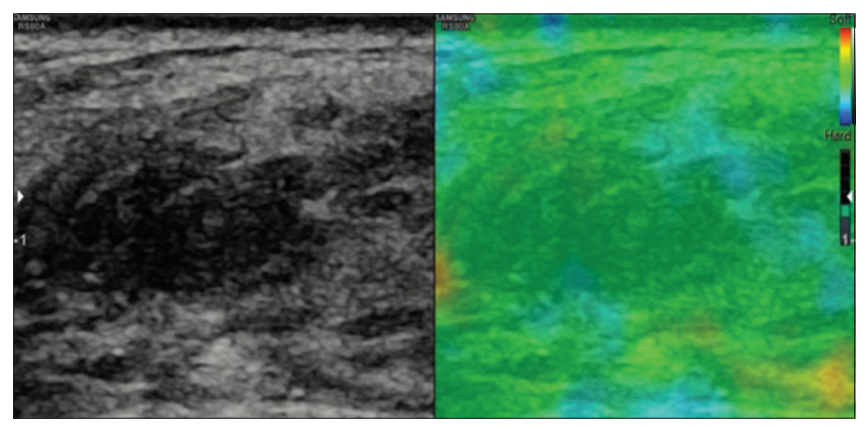

Figure 1: A case of fat necrosis showing strain in entire lesion as represented by even shading of the lesion in color green on elastography suggesting score 1

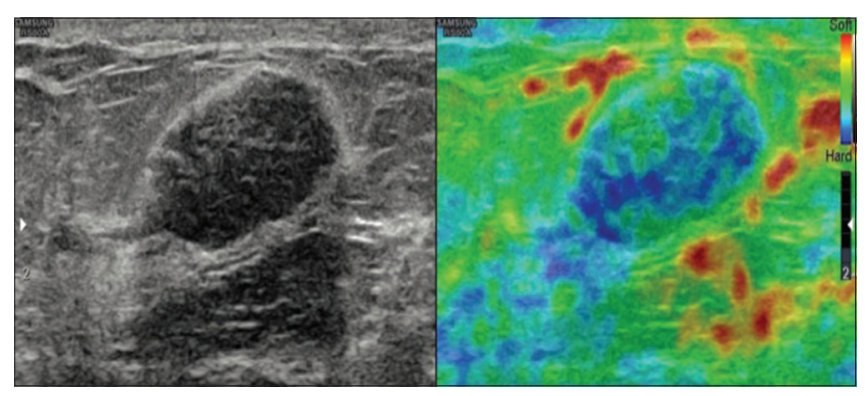

Figure 3: A case of fibroadenoma showing mosaic pattern of green and blue color suggesting a score of 3

Histopathology

Finally, the lesions were subjected to either ultrasound guided core biopsy or surgically excised. Histopathological results were used as the reference standard for comparison of conventional ultrasound and elastography findings.

\section{Statistical analysis}

The sonographic and elastographic parameters for benign and malignant lesions were compared relative to the histopathological diagnosis using the Mann-Whitney $\mathrm{U}$ test. The level of significance was set at a $P$ value of 0.05 . The receiver operating curve (ROC) analysis was used to determine the optimal threshold, area under the curve (AUC), specificity and sensitivity of the tested parameters. Statistically significant differences between the areas under the ROC were reported as $95 \%$ confidence intervals. ROC curves were compared by using the deLong test. Statistical analysis was performed using statistical software version 3.6.0 (core team, 2019).

\section{Results}

We included 100 women with 113 breast lesions. There were $73(64.6 \%)$ benign and $40(35.4 \%)$ malignant lesions. The mean age for benign lesions was 39.9 and 55.8 years for malignant lesions (age range 16-80) [Table 1]. The malignant lesions showed higher ES and SR and a higher BIRADS category as compared to benign lesions $(P<0.001)$ [Table 1]. The median ES for benign lesions was 2, and for malignant lesions it was 5 . The mean SR was 1.82 for benign and 4.67 for malignant lesion.

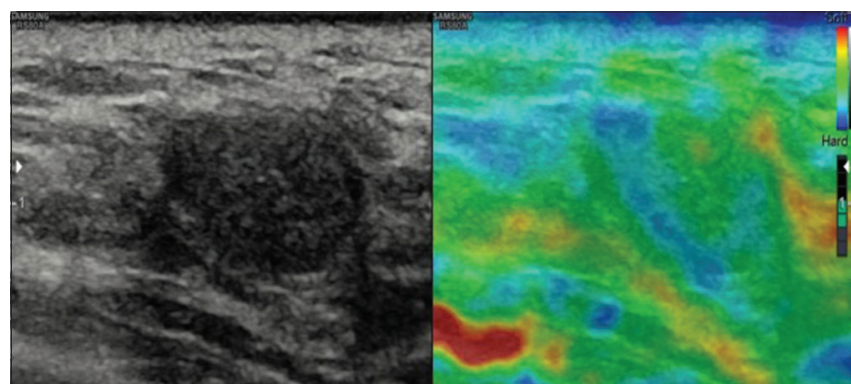

Figure 2: A case of fibroadenoma showing strain in most of the lesion represented by green color with part of the lesion showing no strain, shaded in blue with a score 2

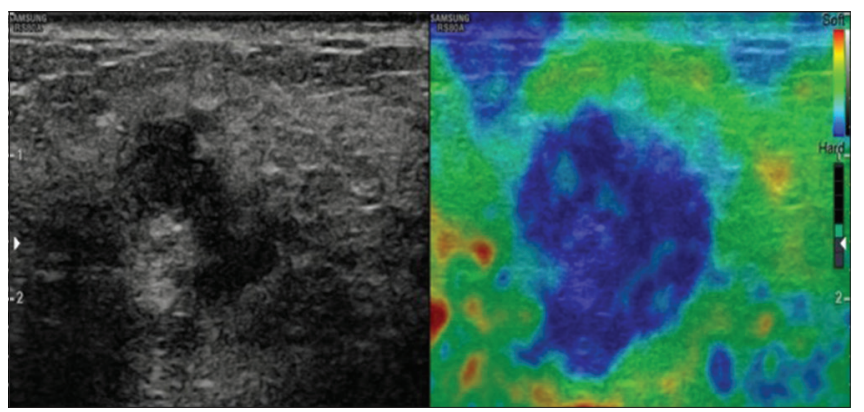

Figure 4: A case of infiltrating carcinoma showing stiffness in the entire lesion represented by even shading with blue suggesting a score of 4

Table 1: Mean values of variables with respect to histopathological diagnosis

\begin{tabular}{lccccc}
\hline Variants & Mean & SD & Median & IOR & $P$ \\
\hline Age & & & & & \\
$\quad$ Benign & 39.97 & 10.81 & 40 & 13.5 & $<0.001$ \\
$\quad$ Malignant & 55.87 & 14.69 & 58 & 22.5 & \\
BIRADS & & & & & \\
$\quad$ Benign & 3.37 & 0.49 & 3 & 1 & $<0.001$ \\
$\quad$ Malignant & 4.65 & 0.66 & 5 & 0.25 & \\
ES & & & & & \\
$\quad$ Benign & 2.42 & 0.62 & 2 & 1 & $<0.001$ \\
$\quad$ Malignant & 4.67 & 0.62 & 5 & 0.25 & \\
SR & & & & & \\
$\quad$ Benign & 1.82 & 0.85 & 1.6 & 0.92 & $<0.001$ \\
Malignant & 4.67 & 1.31 & 4.65 & 1.32 & \\
\hline
\end{tabular}

ROC analysis for ES showed highest sensitivity (92.3\%) and specificity (94.59\%) at cut-off of 3 [Figure 6] with AUC being 0.98 . The positive predictive value (PPV), negative predictive value (NPV) and accuracy were 90, 95.8, and $93.8 \%$, respectively [Table 2]. For strain ratio, the optimal cut-off value was 3.0, with a sensitivity of $90 \%$ and specificity of $93.2 \%$ with AUC 0.96 [Figure 6]. We obtained PPV of $87.5 \%$, NPV of $94.5 \%$, and accuracy of $92 \%$ [Table 2] at this cut-off. A cut-off of 3.5 for SR showed lower sensitivity (87\%) but higher specificity (95\%). The sensitivity, specificity, and accuracy of the conventional ultrasound alone were 90, 63.8, and 72\%, respectively, with AUC 0.90 [Figure 6]. PPV was $57 \%$ and NPV was $92 \%$ [Table 2]. The overall 


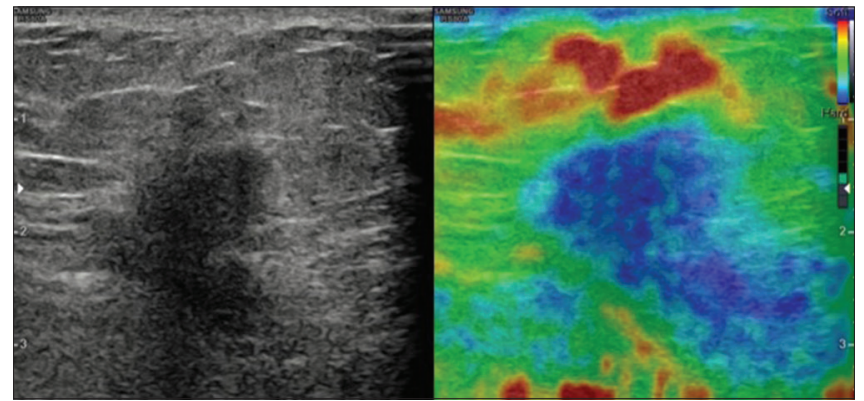

Figure 5: A case of invasive ductal carcinoma showing stiffness in entire lesion as well as in surrounding tissue represented by shading of lesion and surrounding tissue in blue suggesting a score of 5

Table 2: A comparison of sensitivity, specificity, diagnostic accuracy, NPV, and PPV for elastography score, strain ratio, ultrasound, combined elastography score and strain ratio, and combined ultrasound, lastography score, and strain ratio

\begin{tabular}{lccccc}
\hline Parameter & $\begin{array}{c}\text { Sensitivity } \\
(\%)\end{array}$ & $\begin{array}{c}\text { Specificity } \\
(\%)\end{array}$ & $\begin{array}{c}\text { Diagnostic } \\
\text { accuracy }(\%)\end{array}$ & $\begin{array}{c}\text { PPV } \\
(\%)\end{array}$ & $\begin{array}{c}\text { NPV } \\
(\%)\end{array}$ \\
\hline ES & 92.5 & 94.5 & 93.81 & 90 & 95.8 \\
SR & 90 & 93.24 & 92 & 87.8 & 94.4 \\
US & 90.24 & 63.89 & 72 & 57 & 92 \\
ES+SR & 92 & 93 & 92.9 & 88.3 & 95.7 \\
US+ES+SR & 95 & 94 & 94.69 & 90.4 & 97.1 \\
\hline
\end{tabular}

sensitivity and specificity of SE combining both ES and SR were 92 and 93\%, respectively [Table 2]. The sensitivity, specificity, and accuracy of conventional ultrasound and SE combined together were 95, 94, and $94.69 \%$, respectively. PPV was $90.48 \%$ and NPV was $97.18 \%$ [Table 2].

There was a statistically significant difference in the AUC for ES and conventional US (difference between areas $=0.08$, 95\% confidence interval $[\mathrm{CI}]: P=0.011$ ) [Figure 6]. The AUC for ES and SR (difference between areas $=0.02$, $95 \%$ CI: $P=0.17$ ) and conventional US and SR (difference between areas $=0.06,95 \% \mathrm{CI}: P=0.075)$ did not differ significantly [Figure 6].

Out of the 113 lesions, 62 lesions were classified as BI-RADS 4 by conventional ultrasound features. Among these, 36 were found to be malignant and 26 benign on histopathological examination. On the basis of elastography parameters, $22(85 \%)$ lesions were correctly predicted as benign with overall sensitivity, specificity, and diagnostic accuracy of elastography being $97,84.6$, and $91.9 \%$, respectively.

Of 51 lesions classified as BIRADS 3 by conventional ultrasonography, 4 were found to be malignant and 47 benign on histopathological examination. Among these, $97 \%$ were correctly predicted as benign and $50 \%$ lesions were correctly predicted as malignant based on elastography parameters (ES and SR) with a sensitivity of $50 \%$, specificity of $97.8 \%$, and diagnostic accuracy of $94 \%$. The PPV for malignancy was $66.6 \%$ and NPV was $95.45 \%$.

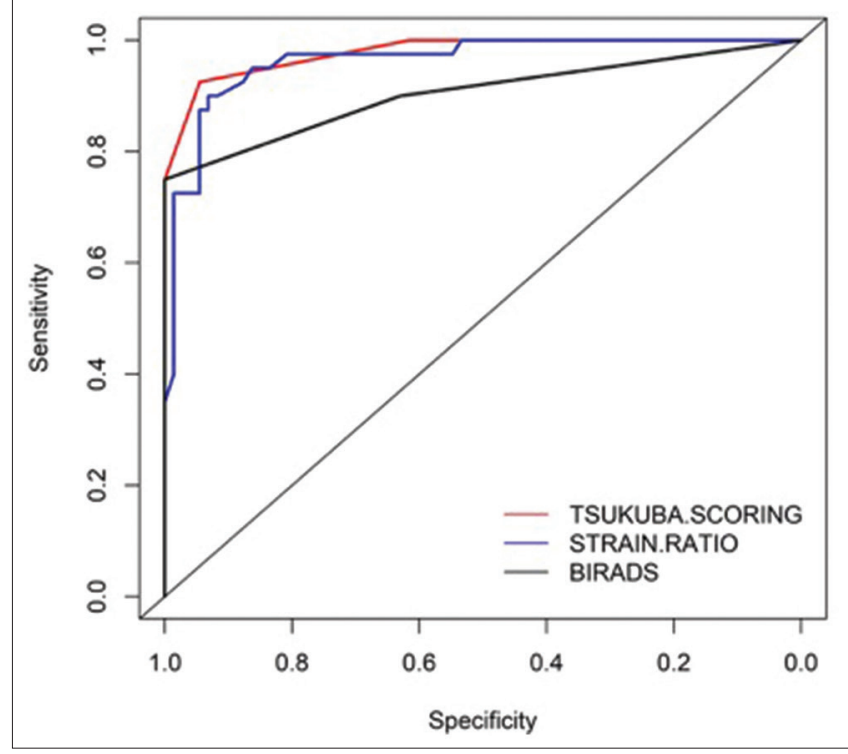

Figure 6: Combined ROC for ES, SR, and US showing respective AUC. AUC showed significant difference between US and ES

The distribution of lesions according to histopathological findings is outlined in Table 3 . The most common malignant lesion was invasive ductal carcinoma $(80 \%)$, and the most common benign lesions were fibro-adenomas $(79.5 \%)$ followed by benign fibro-epithelial lesions (9.6\%). A comparison of overall true positive, true negative, false positive, and false negative cases on conventional ultrasound, ES, and strain ratio with respect to histo-pathological diagnosis is highlighted in Table 4.

\section{Discussion}

The concept of ultrasound elastography was first introduced by Ophis et al. in 1991. ${ }^{[4]}$ Elastography is a noninvasive technique that uses the mechanical property of tissue elasticity on external compression to assess stiffness of tissues analogous to clinical palpation. By offering additional information about tissue stiffness, real-time tissue elastography can help in differentiation between benign and malignant disease, thus improving the accuracy of diagnosis of breast cancer. ${ }^{[5]}$ Recently, ultrasound elastography has been incorporated into the fifth edition of ACR BIRADS lexicon. ${ }^{[6]}$ Bojanic et al., concluded that SE can be used to upgrade or downgrade BIRADS category of breast lesions. ${ }^{[7]}$ Currently, two types of elastography technique are used in clinical ultrasound systems - strain and shear wave. Each technique has its own advantages and disadvantages.

The role of SE in breast imaging has been investigated since 2005. While most of the studies have concluded in favor of elastography over conventional ultrasound, Kumm et al., and Yilmaz et al., reported lower sensitivity and specificity for sono-elastography as compared to conventional ultrasound..$^{[8,9]}$ 
Sinha, et al:: Role of strain elastography for reclassification of indeterminate breast lesions

Table 3: Histo-pathological differential diagnosis amongst malignant and benign lesions

\begin{tabular}{|c|c|c|c|}
\hline \multicolumn{2}{|l|}{ Malignant lesions } & \multicolumn{2}{|c|}{ Benign lesions } \\
\hline Histopathological diagnosis & Numbers Total $=40$ & Histopathological diagnosis & Numbers Total $=73$ \\
\hline Invasive ductal carcinoma & $27(67.5 \%)$ & Fibroadenoma & $58(79.5 \%)$ \\
\hline Invasive mucinous carcinoma & $4(10 \%)$ & Benign fibroepithelial lesion & $7(9.6 \%)$ \\
\hline Invasive poorly differentiated carcinoma & $2(5 \%)$ & Fibrocystic disease & $6(8.2 \%)$ \\
\hline Invasive carcinoma with Apocrine differentiation & $1(2.5 \%)$ & Sclerosing adenosis & $2(2.7 \%)$ \\
\hline Invasive carcinoma with plasmacytoid differentiation & $1(2.5 \%)$ & & \\
\hline DCIS & $4(10 \%)$ & & \\
\hline Malignant Phylloides tumor & $1(2.5 \%)$ & & \\
\hline
\end{tabular}

Table 4: Comparison of true positives, false positives, true negatives, and false negatives among ES, SR, and US with respect to histopathological diagnosis

\begin{tabular}{lcccc}
\hline Parameters & $\begin{array}{c}\text { True } \\
\text { positive }\end{array}$ & $\begin{array}{c}\text { True } \\
\text { negative }\end{array}$ & $\begin{array}{c}\text { False } \\
\text { positive }\end{array}$ & $\begin{array}{c}\text { False } \\
\text { negative }\end{array}$ \\
\hline US & 36 & 46 & 27 & 4 \\
ES & 37 & 69 & 4 & 3 \\
SR & 36 & 68 & 5 & 4 \\
\hline
\end{tabular}

We analyzed two of the most widely studied SE parameters in our study - the ES and the strain ratio SR. Our results showed comparable sensitivity for ES, SR, and conventional ultrasonography. However, elastography (ES and SR) showed better diagnostic performance with high specificity, diagnostic accuracy, and NPV as compared to conventional ultrasound which is in concordance with other studies. ${ }^{[5,7,10-17]}$

Combined use of ultrasound features and elastography parameters (ES and SR) yielded better results than individual parameters in each category in agreement with some of the previous studies. ${ }^{[7,10,11]} \mathrm{Kumm}$ et al., suggested that NPV of a diagnostic test should approach 0.98 to confidently characterize a breast lesion as benign. ${ }^{[8]}$ With combined use of US, ES, and SR, a NPV of 0.97 was obtained in our study.

Wojcinski et al., analyzed BIRADS 3 lesions with sono-elastography and suggested that these lesions can be categorized into low-risk and high-risk groups based on the ES. ${ }^{[18]}$ Our study showed similar results. Among BIRADS category 3 lesions, we correctly predicted benignity in 47 out of 51 lesions owing to their elasticity. In addition, two well-circumscribed malignant lesions, misclassified as BIRADS 3 on conventional ultrasonography, were correctly characterized on elastography owing to a high elasticity score and strain ratio and were thus upgraded to category 4. Among BIRADS 4 category, we were able to successfully downgrade about 22 out of the total 26 histologically benign lesions to BIRADS 3 category, incorrectly characterized as probably malignant on conventional ultrasonography descriptors. In addition to that, five of 4 a category lesions were correctly upgraded to $4 \mathrm{~b}$ or $4 \mathrm{c}$ based on elastography analysis, thereby increasing the diagnostic confidence. These results are in accordance with a metanalysis conducted by Sadigh et al., which indicated that ultrasound elastography has the potential to improve diagnostic accuracy of US. ${ }^{[1]}$ They concluded that in low-risk groups, elastography should be performed in positive ultrasonographic results to avoid unnecessary biopsies. Other studies also support these results with similar conclusions. ${ }^{[7,11,13,16]}$

Apart from assessing the overall performance of elastography, we also tried to compare the individual performance of ES and SR against US, although a qualitative parameter ES performed significantly better than SR or US in distinguishing between benign and malignant lesions, which is similar to Bojanic et al. and Yerli et al.'s findings. ${ }^{[7,19]}$ AUC for ES showed statistically significant difference from conventional ultrasound, with higher specificity, sensitivity, and NPV. We also found that the ES of $>3$, i.e., score $=4$ or 5 has the maximum sensitivity and specificity for the detection of malignancy.

SR is a semiquantitative parameter for measurement of stiffness in a lesion. ${ }^{[20]}$ There have been different opinions among the researchers on the accuracy of strain ratio. According to some studies, SR is more effective and objective parameter for characterization of breast lesions than ES. ${ }^{[13-15,21,22]}$ However, other studies have reported poor reliability and reproducibility of SR and have found it to be less accurate. ${ }^{[8,19]}$ Yerli et al., concluded that after elastographic score, strain ratio is not needed for characterization of breast lesions. ${ }^{[19]}$ In our study, the AUC for SR and US did not show any significant difference. Nevertheless, SR was found to be more specific with high NPV than US alone (93\% specificity vs 63\%).

Furthermore, different studies have shown variable cut-off values for SR for differentiation of benign versus malignant lesions as described in Table 5. We found maximum sensitivity and specificity at a cut-off value of 3 (sensitivity $=90 \%$ and specificity $=93 \%$ ). A cut-off of 3.5 showed higher specificity of $95 \%$. This variation in cut-off value across studies may be attributed to technical factors in acquiring elastography data. Barr et al., described precompression as the major limiting factor in obtaining accurate results with both strain and shear wave 
Table 5: Various studies showing different cut-off values for strain ratio

\begin{tabular}{lcc}
\hline Study & Year & SR Cut off value \\
\hline Thomas et al. & 2010 & 2.45 \\
Gheonea et al. & 2011 & 3.65 \\
Barr et al. & 2012 & 4.80 \\
Alhabshi et al. & 2013 & 5.60 \\
Liu et al. & 2014 & 4.15 \\
Menezes et al. & 2015 & 4.72 \\
Bojanic et al. & 2017 & 3.50 \\
Yilmaz et al. & 2017 & 4.25 \\
\hline
\end{tabular}

elastography. ${ }^{[23]}$ They found that precompression can increase the overall stiffness of the part being examined with more effect on background fat, thereby reducing the strain ratio and yielding false negative results. For correct measurement of strain ratio, minimum precompression should be applied.

The second factor could be inconsistent placement of the ROI. Selection of equal-sized ROI and its placement at the same depth in the lesion and adjacent fat is another important factor for the accuracy of the reading. A third factor is the optimal level of external compression while acquiring elastography data that are imperative for accuracy of results. As it is an operator-dependent process, it can lead to variable SR values and thus can cause interobserver variations.

In addition to the technical factors, histopathological makeup of the breast lesions can also influence the ES and SR values, ${ }^{[24]}$ contributing to the false-positive or false-negative results on elastography. In our study, there were 27 false-positive lesions on conventional ultrasonography, 5 on strain ratio, and 4 on ES [Table 4]. Among these false-positive lesions were two fibroadenoma, one sclerosing adenosis, one sclerosing fibroadenoma, and one benign fibro-epithelial lesion with sclerosing background. In our study, some of the benign lesions with a significant amount of fibrosis showed high ES $>3$ and high strain ratios above our cut-off value 3 , thereby leading to false-positive results. False-negative results on ES was 3 and SR was 4 [Table 3]. Among the false-negative results, two were ductal carcinoma in situ, one was mucinous, and one was invasive ductal carcinoma. They had elasticity score 2 or 3, and SR ranging between 2 and 3. Low ES (2-3) and strain ratio was seen $(<3)$ in some malignant lesions due to inherent softness with no or minimal scirrhous reaction as in mucinous carcinoma.

Our study had some limitations. First is technical. Acquisition and interpretation of SE data are operator dependant leading to interobserver and intraobserver variations, which were not analyzed in our study. Secondly, diagnostic performance of elastography is also affected by the lesion size as suggested by some studies. ${ }^{[7,25]}$ We did not analyze the performance of elastography with respect to lesion size. And finally, the quality of the elastography map depends on the overall breast density and architecture. ${ }^{[26]}$ This factor was not evaluated in our study. We propose that these factors should be assessed in larger studies so that SE may be made more quantitative and reproducible.

In conclusion, SE is a useful adjunct to conventional B mode ultrasound in characterization of breast lesion. The combined use of SE and conventional ultrasound can be used to downgrade a number of BIRADS 3 and 4 category lesions, thereby avoiding unnecessary biopsies and reassuring the physicians for interval follow-ups. Additionally, some of the malignant lesions can only be picked upon elastography based on their stiffness and can help in upgrading the lesions to a higher category for biopsies instead of regular follow-up. ES is the most useful predictor of benignity in breast lesions.

Financial support and sponsorship

Nil.

\section{Conflicts of interest}

There are no conflicts of interest.

\section{References}

1. Bray F, Ferlay J, Soerjomataram I, Siegel RL, Torre LA, Jemal A. Global cancer statistics 2018: GLOBOCAN estimates of incidence and mortality worldwide for 36 cancers in 185 countries. CA Cancer J Clin 2018;68:394-424.

2. Malvia S, Bagadi S, Dubey U, Saxena S. Epidemiology of breast cancer in Indian women: Breast cancer epidemiology. Asia-Pacific J Clin Oncol 2017;13:289-95.

3. Itoh A, Ueno E, Tohno E, Kamma H, Takahashi H, Shiina $\mathrm{T}$, et al. Breast disease: Clinical application of us elastography for diagnosis. Radiology 2006;239:341-50.

4. Ophir J, Céspedes I, Ponnekanti H, Yazdi Y, Li X. Elastography: A quantitative method for imaging the elasticity of biological tissues. Ultrason Imaging 1991;13:111-34.

5. Moon WK, Chang SC, Huang CS, Chang RF. Breast tumor classification using fuzzy clustering for breast elastography. Ultrasound Med Biol 2011;37:700-8.

6. Fleury Ede F. The importance of breast elastography added to the BI-RADS ${ }^{(} 5^{\text {th }}$ ed.ition) lexicon classification. Rev Assoc Med Bras (1992) 2015;61:313-6.

7. Bojanic K, Katavic N, Smolic M, et al. Implementation of elastography score and strain ratio in combination with B-mode ultrasound avoids unnecessary biopsies of breast lesions. Ultrasound Med Biol 2017;43:804-16.

8. Kumm TR, Szabunio MM. Elastography for the characterization of breast lesions: Initial clinical experience. Cancer Control 2010;17:156-61.

9. Yilmaz E, Y1lmaz A, Aslan A, Inan I, Evren MC, Tekesin K. Real-time elastography for differentiation of breast lesions. Pol J Radiol 2017;82:664-9.

10. Menezes R, Sardessai S, Furtado R, Sardessai M. Correlation of strain elastography with conventional sonography and FNAC/ Biopsy. J Clin Diagn Res 2016;10:TC05TC10. 
11. Gheonea IA, Stoica Z, Bondari S. Differential diagnosis of breast lesions using ultrasound elastography. Indian J Radiol Imaging 2011;21:301-5.

12. Barr RG, Destounis S, Lackey LB $2^{\text {nd }}$, Svensson WE, Balleyguier C, Smith C. Evaluation of breast lesions using sonographic elasticity imaging: A multicenter trial. J Ultrasound Med 2012;31:281-7.

13. Liu XJ, Zhu Y, Liu PF, Xu YL. Elastography for breast cancer diagnosis: A useful tool for small and BI-RADS 4 lesions. Asian Pac J Cance Prev 2014;15:10739-43.

14. Parajuly SS, Lan PY, Yun MB, Gang YZ, Hua Z. Diagnostic potential of strain ratio measurement and a 5 point scoring method for detection of breast cancer: Chinese experience. Asian Pac J Cancer Prev 2012;13:1447-52.

15. Thomas A, Degenhardt F, Farrokh A, Wojcinski S, Slowinski T, Fischer T. Significant differentiation of focal breast lesions: Calculation of strain ratio in breast sono-elastography. Acad Radiol 2010;17:558-63.

16. Faruk T, Islam MK, Arefin S, Haq MZ. The journey of elastography: background, current status, and future possibilities in breast cancer diagnosis. Clin Breast Cancer 2015;15:313-24.

17. Sadigh G, Carlos RC, Neal CH, Dwamena BA Ultrasonographic differentiation of malignant from benign breast lesions: A meta-analytic comparison of elasticity and BIRADS scoring. Breast Cancer Res Treat 2012;133:23-35.

18. Wojcinski S, Boehme E, Farrokh A, Soergel P, Degenhardt F, Hillemanns P. Ultrasound real-time elastography can predict malignancy in BI-RADS (R)-US 3 lesions. BMC Cancer 2013;13:159.
19. Yerli H, Yilmaz ., Kaskati T, Gulay H. Qualitative and semi quantitative evaluations of solid breast lesions by sonoelastography. J Ultrasound Med 2011;30:179.e86

20. Barr RG, Nakashima K, Amy D, Cosgrove D, Farrokh A, Schafer F, et al. WFUMB guidelines and recommendations for clinical use of ultrasound elastography: Part 2: breast. Ultrasound Med Biol 2015;41:1148-60.

21. Yoon SK, Jung GP, Beom SK, Lee CH, Ryu DW. Diagnostic value of elastography using acoustic radiation force impulse imaging and strain ratio for breast tumors. J Breast Cancer 2014;17:76-82.

22. Alhabshi SM, Rahmat K, Abdul Halim N, Aziz S, Radhika S, Gan GC, et al. Semi-quantitative and qualitative assessment of breast ultrasound elastography in differentiating between malignant and benign lesions. Ultrasound Med Biol 2013;39:568-78

23. Barr RG, Zhang Z. Effects of precompression on elasticity imaging of the breast. J Ultrasound Med 2012;31:895-902.

24. Okar Atabey A, Aribal E, Ergelen R, Kaya H. Value of strain elastography ultrasound in differentiation of breast masses and histopathologic correlation. J Breast Health 2014;10:234-8.

25. Giuseppetti GM, Martegani A, Di Cioccio B, Baldassarre S. Elastosonography in the diagnosis of the nodular breast lesions: Preliminary report. Radiol Med 2005;110:69-76.

26. Regini E, Bagnera S, Tota D, Campanino P, Luparia A, Barisone $\mathrm{F}$, et al. Role of sonoelastography in characterising breast nodules: Preliminary experience with 120 lesions. Radiol Med 2010;115:515-62. 